INTERNATIONAL DESIGN CONFERENCE - DESIGN 2018

https://doi.org/10.21278/idc.2018.0496

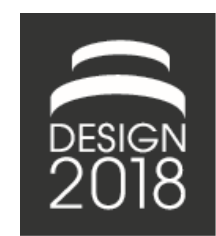

\title{
EVALUATION OF PRODUCT DEVELOPMENT: A COMPARATIVE CASE STUDY
}

\author{
N. Koehler, P. Močibov, T. Naumann and S. Vajna
}

\begin{abstract}
The aim of the paper is to reveal phenomena of integrated design engineering in practice by comparing four different product developments in a case study. Based on our universal questionnaire, the product developments in the branch automotive, sports optics, power tools and camera are analyzed in terms of their abilities in the field of problem solving, complexity and traceability. With the help of the collected data, we confirm well-known product development phenomena such as the ill-definedness of goals, communication as complexity driver and the lack of traceability in design rationale.
\end{abstract}

Keywords: integrated product development, problem solving, complexity, traceability

\section{Introduction}

Today's product development is characterized by a rising complexity. This inherent complexity needs to be handled as a holistic system in order to develop sophisticated products. As technology evolved over time, also the needs of individuals and therefore the needs of the market evolved. Basic needs like the possibility to transport goods from one place to another led to the development of cars in the last century. The more advanced needs such as the ability to travel from one place to another without any human intervention will lead to autonomous transportation in the next decade. In other words, the problems of the future are those which today's products are solving only partially and on the basic level. Future products are supposed to solve same problems with minimal human interaction in the most comprehensive and natural way. In that manner, products evolved from simple mechanical to complex smart products and therefore, the product development needs to adapt as well. In this paper, needed adaption of product development to deal with the increasing product complexity is presented.

For the purpose of universality, four different product developments are investigated including those in automotive, sports optics, power tools and camera branch. To determine the state of the art of the product development, our earlier developed questionnaire is introduced exploring (1) problem solving abilities, (2) complexity not only of products, but also of processes and organization and (3) possibilities of tracing various objects. Since the challenge of understanding the complexity will become even greater in future, we inspect traceability as an approach on how to handle it. Therefore, one of the aims of the questionnaire is to develop a descriptive study evaluating the need for traceability from engineers' point of view. Additionally, demographic information is collected to get a better overview of the context of particular product development.

\section{Theoretical background}

The main purpose is, through the eyes of the engineers, to determine the complexity of the product development of technical systems and to analyze the ability to trace objects from their origin to their use in the product (Koehler et al., 2015). As mentioned, study's main focus is set on complexity and 
traceability during the problem solving process of product development. The main purpose is to determine the perception of each of dimensions through the eyes of engineers. Accordingly, the questionnaire consists of three major parts: (1) the ability of problem solving in product development, (2) the determination of product, process and people complexity, (3) the traceability with respect to objects, subjects, source, tool, design rationale and time. In following paragraphs the three constructs and their items as basis for the questionnaire are explained in detail.

\subsection{Problem solving}

Problem solving as the first dimension of the questionnaire analyzes the ability of product development to solve problems systematically. In order to evaluate the problem solving in any product development environment the problem solving cycle based on Hall (1962) adapted by Haberfellner et al. (2012) for systems engineering is used. Accordingly, the constructs of problem solving distinguish between three activities: problem clarification, solution search and solution selection. With the help of problem solving constructs the main difficulties of engineers solving problems can be detected. Items within these constructs are as follows (Hall, 1962; Haberfellner et al., 2012; Ehrlenspiel and Meerkamm, 2013):

- The problem clarification as the first step of problem solving focuses on the analysis of the initial situation in order to formulate the goals. The situation analysis considers if the initial situation of the problem setting is analyzed sufficiently. Subsequently, the formulation of goals evaluates to what extent the goals are formulated in terms of requirements.

- The solution search as the second step of problem solving comprises the creative synthesis of solutions as well as the crucial analysis of solutions. During the systems synthesis various variants of solutions are generated based on the situation analysis and goals formation. The system's analysis, however, examines the feasibility of the developed solution concepts.

- The solution selection as the third step of problem solving takes the evaluation of the feasible solutions and the selection of the final solution into consideration. The evaluation of solutions compares the different developed solutions based on the requirements generated solution variants. Finally, the optimal solution is selected based on the degree of fulfilment concerning the requirements.

\subsection{Complexity}

The second dimension of the questionnaire investigates complexity of product development from the viewpoint of an engineer. Taking the differentiation between complexity and complicatedness into account, then the subjective evaluation of complexity in a questionnaire represents more complicatedness than complexity. However, considering a holistic point of view along with the concept of collective intelligence presented by Rieckher (1871), then the evaluation represents rather complexity than complicatedness. According to Lindemann et al. (2009) the main constructs for the evaluation of internal complexity are product, process and organizational complexity. Below, the constructs of complexity with their associated items are presented.

- The product complexity of a technical system depends on the variety, the connectivity as well as on the variance of engineering objects. The variety of a technical system refers to the number and types of engineering objects, whereas the connectivity deals with the number and types of engineering object relations (Patzak, 1982; Lindemann et al., 2009; Ehrlenspiel and Meerkamm, 2013). According to Weber (2005) the variance of engineering objects is the third item describing the product complexity.

- The process complexity of the product development depends on the multidisciplinary, the development-cycle time as well as the iterations. Multidisciplinary is connected to the number of involved disciplines to develop a technical system. Development-cycle time is described as the time to develop new solutions. Iterations represent the number of repetition in order to find a solution (Lindemann et al., 2009).

- The organizational complexity of social systems depends on the division of labor, the interaction and the levels of hierarchy. The labor-division is measured by the split of responsibilities for the development of technical systems. Interaction describes the need for coordination between 
developers. The level of hierarchy is determined by the number of organizational levels (Baccarini, 1996).

\subsection{Traceability}

Traceability within the scope of the questionnaire represents a quality factor of designing [Stor-2004]. Various traceability constructs are characterizing the ability to follow engineering objects in product development. The constructs for the evaluation of traceability are based on the trace-ability dimensions proposed by Ramesh and Jarke (2001). These dimensions are described by Winkler and von Pilgrim (2010) as the core questions about engineering objects that can be answered by traceability. The constructs include objects, subjects, sources, tools, rationales and time. For each of those constructs four items of the questionnaire were developed as presented below (Ramesh and Jarke, 2001; Storga, 2004; Winkler and von Pilgrim, 2010; Ouertani et al., 2011):

- What objects need to be traced in product development? Describes the objects that are engineering objects as well as their relations. Engineering objects are all artefacts arising from product development represented by information fragments, e.g. requirements, functions, and components. Engineering object relations are all connections among engineering objects such as aggregation, composition and definition.

- Who are the subjects that need to be traced in product development? Describes the sub-jects that are responsible for creating, modifying and using of engineering objects. Be-sides, subjects make decisions with respect to engineering to develop products effectively and efficiently.

- Where are engineering objects stored as source in product development? Describes the sources for locating engineering objects, e.g. implicit, informal as well as formal sources. Implicit sources refer to people, policies or procedures. Informal sources correspond to requirement specifications, meeting minutes or design documentations. Formal sources re-late to assemblies, part lists or drawings. Finally, required engineering object need to be found to fulfil the objective.

- How are engineering objects used by respective tools in product development? Describes the tools and their functionalities that are used for handling engineering objects and their engineering object relations, e.g. documentation, management, propagation or impact analysis. Documentation and management functionalities focus on the representation of engineering objects. Whereas, propagation and impact analysis intend to describe the change process of engineering objects by the use of the engineering objects relations.

- Why are engineering objects manipulated in product development? Describes the rationale behind creating, modifying and using engineering objects. Especially, the possibility to record decisions formally in order to document the history, document the reasons and estimate the impacts of decisions.

- When are engineering objects handled in product development? Describes the time of creating, modifying and using engineering objects. Timestamps of engineering objects and their engineering object relations are necessary to demonstrate the evolution of the product development. Further, versioning is required to return to past design states as well as to compare current solutions with older once.

\section{Research methodology}

This paper applies a study, earlier developed by Koehler et al. (2015), analyzing product development characteristics across four product developments. The combined sample comprised in total 43 participants with participants from the field of automotive (19), sports optics (12), power tools (12) and camera (19). The applied questionnaire consists of 58 closed-ended questions divided into four parts: problem solving (6), complexity (16), traceability (30) and demographic information (6). Based on the research objective for each part, we developed construct variables which we further divided into the items of the questionnaire maintaining the questionnaire's universality. To measure the attitude of the participants we used a five-point Likert scale distinguishing between "strongly disagree" and "strongly agree". In addition to the closed-ended questions, every part contains one open- ended question offering the possibility to comment that particular part. In order to illustrate the implementation of the 
questionnaire's framework, please find the entire questionnaire in Koehler et al. (2015). As an introduction, in latter paragraphs we first analyze the demographic structure of each of the four samples.

\subsection{The automotive sample}

The automotive sample was drawn from people being responsible for the early automotive product development phase before any physical prototypes are involved. The majority of participants $(0.42)$ stated to be between 45 and 54 years old. About one-quarter of the automotive sample answered to be between 25 and 34 years $(0.26)$ and further one-fifth between 35 and 44 years $(0.21)$. Only ten percent (0.11) declared to be older than the age of 55. Similar applies for the seniority, with half of the participants having between 10 and 19 years of experience. One-third (0.33) of the sample had less than ten years of experience and only the minority of six percent (0.06) had been working between 20 and 29 years. The remaining eleven percent $(0.11)$ is the most senior one with between 30 and 39 years of experience. The majority in the automotive sample answered to have a degree from a university of applied science or bachelor (0.59) and a diploma or master's degree from a university (0.29). Due to the fact that the sample was drawn from the early automotive product development phase, the majority of participants (0.73) were focusing on the product as a whole. Moreover, most of the respondents $(0.89)$ in the automotive sample specialized on mechanical engineering during their education. In favour of that fact speaks that more than eighty percent $(0.89)$ of the respondents come from the development department. Additionally, the automotive sample included no female respondents (0.00). In summary, the automotive sample is characterized by high experienced and slightly older male mechanical engineers.

\subsection{The sports optics sample}

The sports optics sample was collected from people being responsible for the product development in a more integrated way. The majority of the participants $(0.50)$ answered to be in the age between 25 and 34. Subsequently, less participant $(0.25)$ were between 35 and 44 years to a minority of under ten percent $(0.08)$ being older than 54 years. Accordingly, the majority of participants $(0.67)$ said to have less than ten years of experience. However, nearly one-fifth (0.17) answered to have experience between 30 and 39 years. Regarding the educational level, the sports optics sample is characterized by a majority of respondents $(0.75)$ holding a diploma or a master's degree. Moreover, the sports optics sample is more diverse with in total one-third of electrical engineers $(0.17)$, computer scientists $(0.08)$ and businessmen (0.08). Nevertheless, still more than half of the sample (0.67) had a mechanical engineering background. The distribution of tasks differentiates from the automotive sample with a one-third (0.33) of the questioned being involved in developing components, one-fourth $(0.25)$ focusing on project management, and about one-fifth (0.17) each concentrating on system development and requirements management. In line with the automotive sample the majority of the respondents of the sports optics sample were from development division, though one-quarter $(0.25)$ of the participants were female. In total, the sports optics sample represents more an integrated product development setup with more diverse participants.

\subsection{The power tools sample}

The power tools sample was drawn from people responsible for the product development with a focus on mechanical engineering. The majority of the participants $(0.84)$ answered to be between 25 and 44 years old with about half $(0.42)$ being under and half $(0.42)$ being over 35 years of age. A smaller portion (0.16) of the respondents were between 45 and 54 years old. Consistently, the majority $(0.67)$ of the participants had an industry experience of less than 10 years with the rest $(0.33)$ having experience between 10 and 20 years. The education level of the power tools sample is very similar to the sports optics sample with a majority of respondents $(0.67)$ holding diploma degree. Still, the sample contained a quarter of people $(0.25)$ having a bachelor or a degree from a university of applied science. Concerning the specialization during education, the power tools sample is homogeneous with all participants (1.00) having mechanical engineering background. A reason for that is that the sample is drawn from the engineering department only. Accordingly, the majority of the respondents (0.58) are involved in 
component development. The rest of the participants are divided between working on project management (0.17), system development (0.17) and requirements management (0.08). Due to the focus on the engineering nearly all respondents (0.92) are part of development division and a minority $(0.08)$ representing the research division. Regarding the gender, less than ten-percent (0.08) were female participants. The sample from power tools represents a product development with stronger focus on mechanical engineering.

\subsection{The camera sample}

The camera sample describes well the fact that this is a product category which is lately driven by improvements in electronics and software rather than mechanics. Concerning the age structure, most of the participants were between 45 and 54 years $(0.47)$ and between 35 and 44 years $(0.37)$ old. Whereas only around one sixth of respondents were young professionals between 25 and $34(0.11)$ and even younger than 25 years $(0.05)$. Additionally, with a majority $(0.88)$ having less than ten years of experience in that specific company, we see that the seniority of the respondents is comparably low. The sample was drawn from the development department only, resulting in a sample that includes a majority of respondents having an electrical (0.47) and mechanical engineering (0.42) background whereas only minority of the sample specialized in software engineering (0.05) and business (0.05). Regarding the educational level, the sample exhibits a majority $(0.74)$ holding master or diploma degree and a minority holding either a bachelor degree $(0.11)$ or a doctoral degree $(0.16)$. In contrast to the automotive sample, the camera sample includes mainly respondents from the components development $(0.56)$ rather than product management $(0.00)$. Further, each about one-fifth of the participants are involved in project $(0.19)$ and process $(0.19)$ related activities. Concerning gender, the female respondents represent a minority $(0.11)$ of the sample. Overall, camera sample is a comparatively different sample with stronger focus on electrical engineers developing components for the final product.

\section{Results and discussion}

Originally, the study was developed to evaluate the need for traceability from an engineer's point of view. However, the results of the applied study regarding the problem solving and the complexity domain including traceability is of high interest for the research community. Above, each sample was described in terms of demographics, specialization and responsibility of participants illustrating that the four samples represent product development well from an integrated product development perspective. Further analysis focuses on the comparison between the samples for each of the dimensions. For each problem solving, complexity and traceability, mean values of the items and the constructs are used in graphs to present the ability of a sample. In addition, we draw a perceived potential of respondents as a contour across the constructs for each sample to better illustrate trends and patterns. Based on the difference of the ability and potential we discuss the opportunity for improvement on the construct level for each of dimensions.

\subsection{Problem solving}

Problem solving as the first part of the questionnaire analyzes the capability of the respective product development to solve problems systematically along the development-cycle. Investigating the problem solving results of the four samples show novel insights in similarities and differences among diverse industrial product development environments. Figure 1 shows the items of problem solving as well as the average answers across the samples. The results reveal various similarities among the independent samples that might be generalizable for other product developments as well. In the dimension of problem solving, the contour drawn by connecting items' mean values for one sample has a specific form which can be noticed as a pattern that applies to all four samples. This is especially visible in the constructs of problem clarification, items of situation analysis and goal formulation, and the solution search, items of system synthesis and system analysis. Here three patterns can be recognized which we further analyze in detail. 


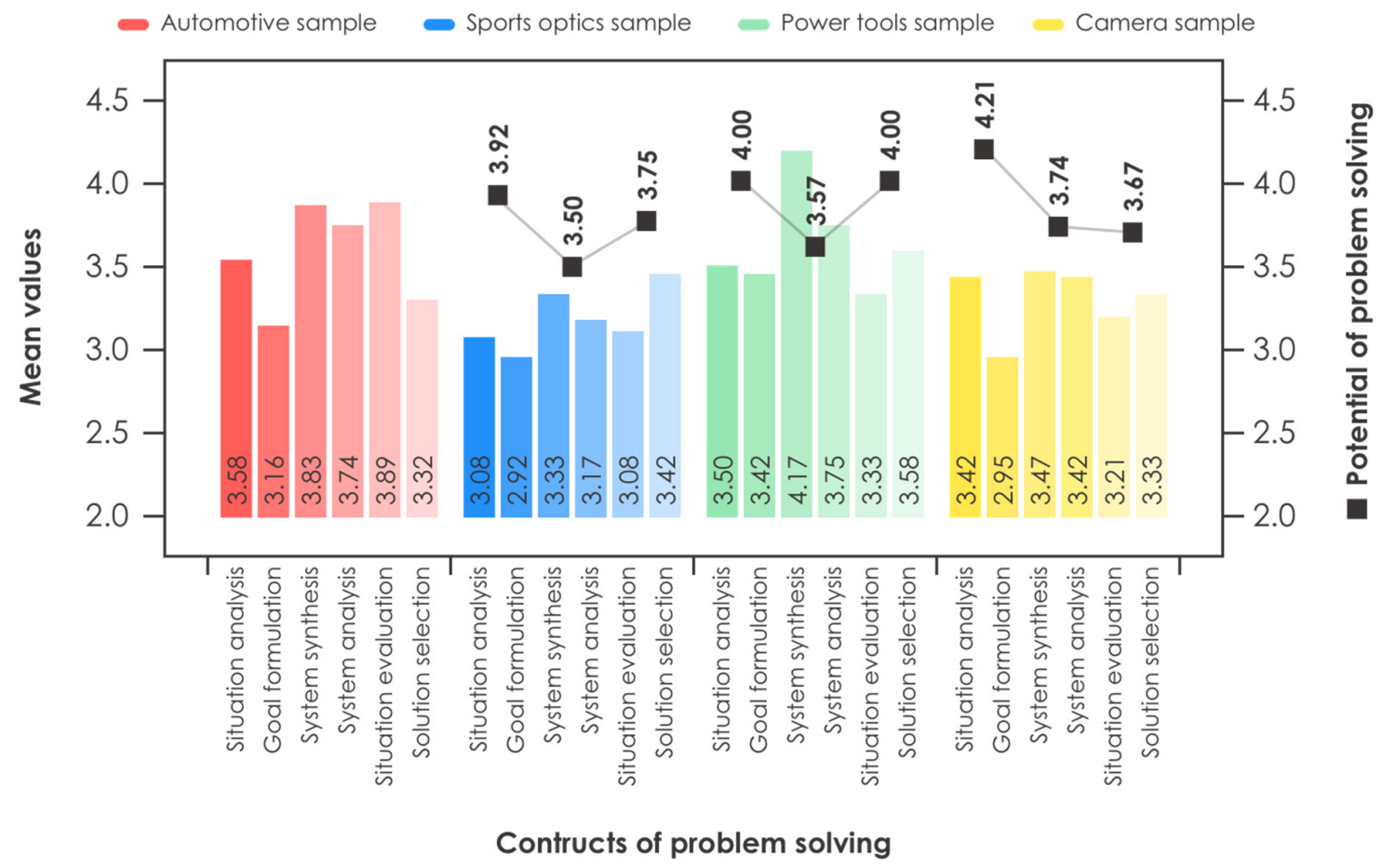

Figure 1. Ability and potential of problem solving

First pattern shows that the ability of goal formulation as second part of the problem clarification is in all samples lower than the situation analysis. Moreover, the goal formulation is the item with the lowest mean value for the automotive, sports optics and camera sample and the second lowest for the power tools sample. Preceding indicates that, from the point of view of the people involved in product development, the desired final state is somewhat unclear. This detected phenomenon is an evidence for the so called "fuzzy front-end" of product development that is characterized by undefined and uncertain goals (Clarkson and Eckert, 2005). Andreasen et al. (2015) are arguing that "ill-definedness [of goals] describes the fact that design essentially concerns products that do not exist yet". We can say that results of the four samples support the hypothesis of Maier and Störrle (2011) that engineering design problems are ill-defined and "precise goals are unknown at the start". Moreover, samples confirm the statement of Eder and Hosnedl (2010) that for design problems the "undefined solutions are the norm. However, the importance of a precise goal formulation in product development is stated by Hall (1962) "it is much more important to choose the "right" objectives than the "right" system". Therefore, it stays as a point for improvement to clarify the undefined goals in the fuzzy front end better.

As second pattern we noticed that the system synthesis as part of the solution search is in all samples higher than the goal formulation and solution analysis. Besides, the solution synthesis is the item with the highest mean for the power tools and camera sample and the second highest mean for both automotive and sports optics sample. This shows that for the people involved in the different product developments, the creation of alternative solutions is sufficient. The high agreement on the system synthesis is backed up by Hashemi Farzaneh et al. (2012). An experiment showed that during the solution search a numerous number of partial solution alternatives are generated. However, only a few "documented solution ideas remain" at the end of the solution search phase. Additionally, although the agreement on system analysis is not as high as on system synthesis it is still for all the samples among the highest values among all the items. This can be recognized as a third pattern. With high values in both system synthesis and system analysis, we can draw a parallel to a high level of iterations during the solution search. According to Maier and Störrle (2011), iteration is a "characteristic of all creative activities [...] by nature". An additional study has shown that this characteristic "enjoys by far the strongest support" (Maier and Störrle, 2011). Bearing earlier in mind, it is understandable that the results 
show solution search and especially the system synthesis is perceived as being sufficient for the product development.

In addition to the patterns with similarities across the four samples the following part is examining each sample with comparison to the others according to Figure 1. First, the automotive sample has an average problem solving ability with two strong outliers in goal formulation and solution selection. The automotive product development is the most complex of the three and therefore enables less transparency on the goals formulation and solution selection for each individual involved. However, the solution evaluation in such a complex environment needs to be comparatively formal step as basis for decision making. Second, the sports optics sample is characterized by the lowest ability of problem solving almost at all items apart from solution selection. One reason for the lowest ability of the product development might be that it is the smallest and therefore has the least structured problem solving approach. However, being the smallest might also lead to a higher transparency for the solution selection. Third, the power tools sample has the highest ability of problem solving across the most items. Especially, the solution search has the highest ability across the four samples. A reason for this might be the sample's strong focus on mechanical engineering as well as the complex dynamics of the product. The high level of uncertainty due to the dynamism of the product might also lead to the lowest value in solution evaluation. Fourth, the camera sample shows the second lowest ability of problem solving with similar characteristics as the sports optics sample. With the ability of situation analysis as the significant difference. In total, the ability of problem solving across the four samples demonstrates distinct similarities with smaller variations in details.

Apart from detecting the current ability of the product development on each problem solving step, Figure 1 shows the comparison of sports optics, power tools and camera samples concerning the opportunity to improve that ability. Corresponding to the ability of problem solving, the comparison of potential shows many similarities among the samples. First, for all three product developments the contour of the graph is describing the potential to enhance the problem solving capabilities that are reverse to the contour describing the abilities. Being the difference of ability and potential, the opportunities for improvements show the same reverse characteristic. Even though the potentials of the power tools samples are evaluated higher, the resulting opportunity to improve is lower. Therefore, to improve the capability of the problem solving, the power tools product development should focus on solution selection and problem clarification. Same is for camera sample with an exception that an overall perception of potential in solution selection is rather low compared to other two samples. Similar to camera and unlike power tools sample, the sports optics sample inherits a positive opportunity to enhance problem solving for every item, with the highest opportunity in the problem clarification. The automotive sample is lacking in this comparison because the value of this type of self-assessment was noticed only after the first application of the questionnaire. By analyzing the potential of other dimensions, we realized that it provides a strongly needed recommendation on where to put the focus and effort for improvements. Therefore, for further samples, we incorporated the set of questions about the potential for problem solving.

\subsection{Complexity}

The complexity according to the three complexity domains people, process and product are researched subsequently. The results based on the four samples reveal important insights regarding differences and similarities among developments of different product. Figure 2 simplifies the complexity analysis to the three constructs: product, process and people complexity. The figure illustrates that for every sample a different complexity construct is dominant. For the automotive sample the product complexity is by far the most dominant with its items i.e. variety, connectivity and variance. In contrast, the sports optics as well as camera sample has the highest perceived complexity for the people domain with interactivity being the dominant item. The power tools' complexity is characterized by a high process complexity due to perceived short development-cycles. Additionally, the major similarity is hidden on the item level not shown in Figure 2. One of the characteristics over all four samples is that the item of multidisciplinary is relatively high. As the opposite, hierarchy is perceived to be relatively low in all examined product developments. Further, the "need for coordination between developers" is one of the highest for all four samples. Latter is in line with current research, e.g. Maier et al. (2011) is stating that 
"communication is one of the critical factors in [...] complex product development". Furthermore, Gopsill et al. (2013) found that "communication is fundamental to engineering design and supports almost all engineering design activities". In total, although each of the samples is driven by different complexity construct, the analysis showed the most similarities within the items of the people domain. Considered that the interactivity is the overall main driver on the item level it is clear that in nowadays product development people are getting more important than ever before (Vajna, 2014).

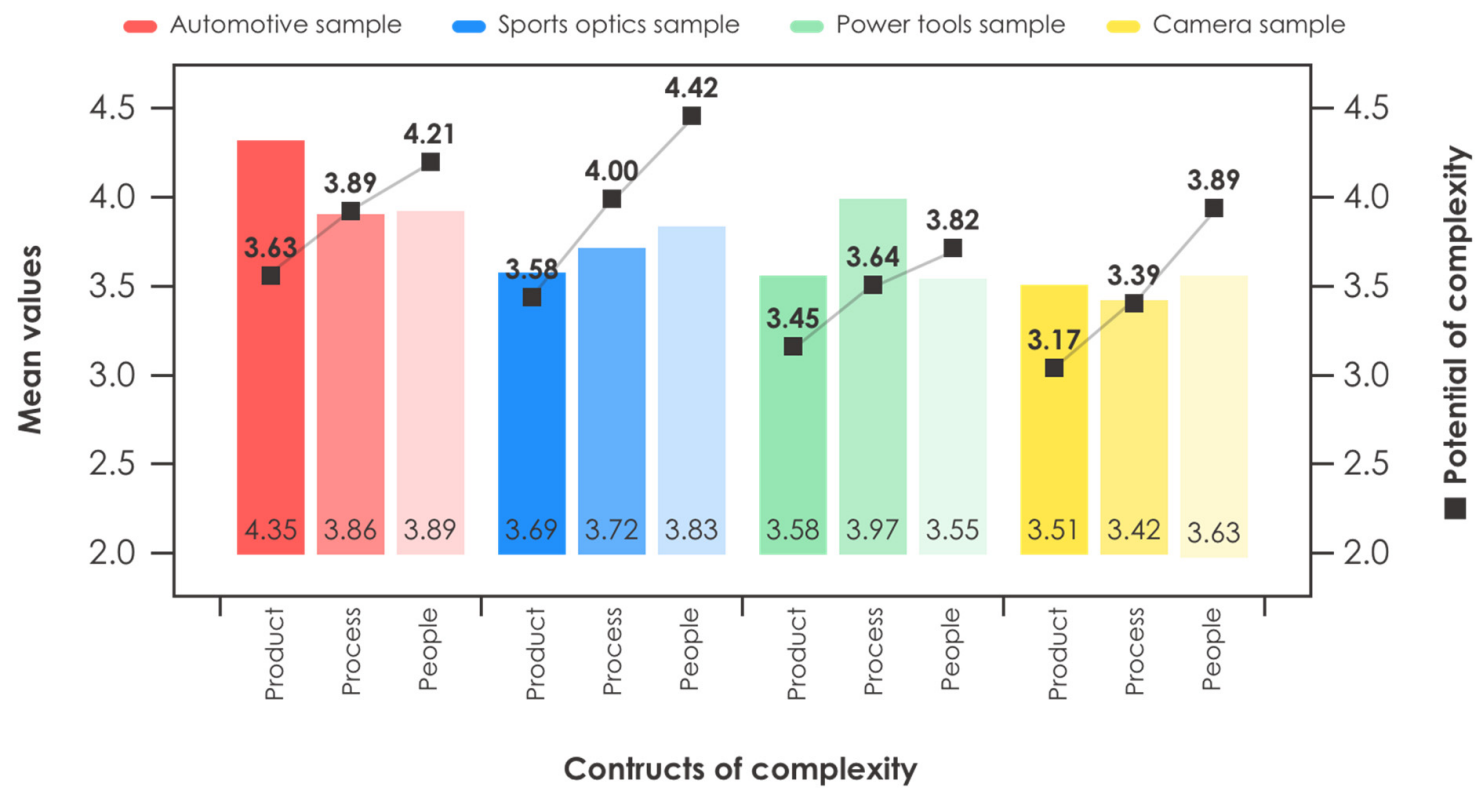

Figure 2. Perceived complexity and potential

In addition, combining the four samples on the construct level shows an equivalence of the three complexity domains. This not only supports the equivalence principle between the complexity of social system and the complexity of the developed technical system (Naumann et al., 2011; Naumann and Koehler, 2014; Naumann, 2016), but also extents it with the complexity of the people domain. Maurer's (2007) existing research on structural complexity is going in the same direction that "a mutual connectivity is the reason why considering only the isolated aspects of complexity is often misleading". Conspiring, Kallenborn (2013) argues that "process complexity is a result of product complexity and is embedded in organizational complexity". Additionally, according to Lindeman et al. (2009), the product complexity, process complexity and people complexity is driven by the market complexity. With constructs and items as they are, the questionnaire is able to uncover complexity imbalances in product development. However, one of the limitation of the research is that, to confirm Lindeman's thesis, an external analysis of the market complexity is needed. Nevertheless, by revealing the imbalances, e.g. the higher product complexity of the automotive sample or the higher process complexity of the power tools sample, and discovering perceived potential for improvement, the questionnaire is able to suggest which domains need to be adapted to gain a more balanced product development.

As mentioned, the questionnaire offers the possibility to detect the perceived potential for complexity across all samples on construct level. Figure 2 shows the consistency of the four samples unveiling two patterns: (1) the potential of people complexity is the highest followed by process and product complexity and (2) the potential of product complexity is for all four samples lower than the perceived product complexity. First, the participants for each sample see the biggest potential and opportunity in "supporting of collaboration in product development". A reason for this high potential on people complexity might be the need to handle the high complexity of the "coordination between developers" mentioned before. Subsequently, the second highest potential across the samples is the process complexity, which is pushed especially due to the high multidisciplinary and the short development 
cycles. Besides, the product complexity potential is the lowest across all samples. Although, the participants perceive the product complexity as the highest, they see restricted potential in "investigating new product development methods". Moreover, the product domain has no opportunity to handle complexity because for each sample the potential is lower than the perceived complexity. Generally speaking, to handle the product complexity in future product development better, more than only new methods is needed.

\subsection{Traceability}

Traceability as the third dimension of the study evaluates the ability to trace objects during the product development. Figure 3 illustrates the traceability abilities of the four samples on the construct level and presents the identified potential. Although traceability is being discussed increasingly also in industry, first obvious observation is that current abilities to trace objects are still neither equal nor sufficient in all four examined product developments. The ability of traceability across all constructs is the highest for the power tools sample followed by the automotive and sports optics sample. Therefore, the product life cycle systems landscape of a specific product development seems to make a difference on the abilities of traceability. Second, having a closer look at the characteristic of the traceability constructs of the power tools sample uncovers the same characteristics as the automotive and camera sample. This might lead back to a similar systems landscape of the product development. Third, comparing the sports optics ability of traceability with the other three samples exhibit the lowest ability with a slightly different characteristic. The two main differences are the comparatively lower ability to trace both objects and time. However, apart from that lack of peaks in object and time traceability constructs for sports optics sample, all samples show almost equal characteristics each with the different average level of constructs indicating the implementation's maturity of the used traceability system. Fourth, across all four samples, it can clearly be identified that there is the biggest gap in the ability to trace rationale. Following is the tool traceability with the second lowest agreement of the respondents in all of the samples apart from camera. The source traceability is, however, relatively sustainable for all samples. In addition, the power tools and automotive sample have a well-balanced ability of time and object traceability. In total, it is clear that in all samples the traceability level is not satisfactory and needs to be improved especially in the field of rationale.

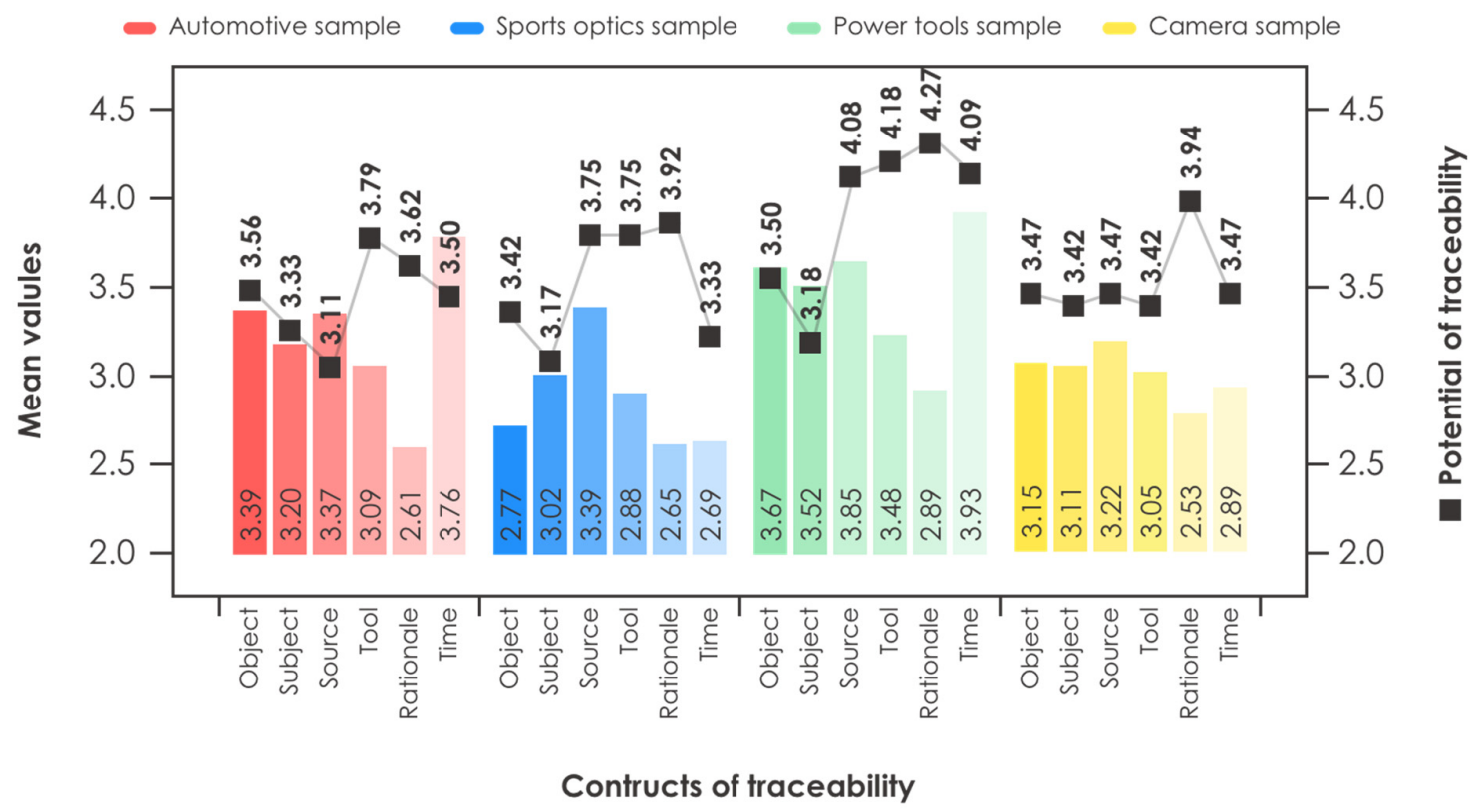

Figure 3. Ability and potential within traceability 
The analysis of the traceability potential gives even more insights which traceability constructs are worth improving. First, as illustrated in the Figure 3, even though the power tools sample already possesses the highest ability of traceability, the highest potential is seen by the respondents in exactly that sample. This leads to the hypothesis that a superior ability of traceability enables to see the potential of the approach. In contrast, the ability for the sports optics and camera sample is comparatively low, however, the participants see a high potential. Second, the characteristic of the traceability potential across the constructs is of special interest. In analogy to problem solving and complexity, the priorities to enable traceability are independent from the product development environment. This is very much visible by comparing the traceability potential of the power tools and the sports optics sample. Both, show the same characteristics with rationale traceability having the highest and the subject traceability the lowest potential. However, also the automotive and camera sample show similar characteristic with slightly different priorities on tool and source traceability. Third, considering the opportunity to improve traceability as the difference between the ability and the potential, quite distinct picture is visible. Across all the samples the ability of rationale traceability is the lowest with nearly always the highest potential is forming the largest opportunity to improve - followed in most cases by the tool traceability. Both are well-known shortcomings addressed by current research directions on tools for "traceability in system engineering" Königs et al. (2012) and "supporting traceability of design rationale" Elgh and Poorkiany (2012). Moreover, the source and time traceability have the lowest incentive to be improved to enable better traceability in product development. Over all, the analysis of the traceability potential confirmed the insight by analyzing the traceability ability especially for rationale and tool traceability.

\section{Conclusion}

The aim of the paper was to evaluate problem solving, complexity and traceability in order to reveal common phenomena of integrated design engineering. Therefore, an earlier developed universal questionnaire was applied to four different product development environments in a case study. The results showed quantitative insights in product development in practice. First, during the problem solving the four samples showed a lack in goal formulation in practice, also known as the fuzzy frontend. Second, the results showed that the human-centric interactivity is the main driver for complexity in product development. Third, the main opportunity to improve traceability in product development is to improve the rationale and tools. In summary, the results of the case study are a substantial contribution to quantify well known design phenomena in industry practice.

The main limitation of the paper is the limited number of participants for each sample and the restricted evidence for reliability of the constructs. Even though the total number of respondents were adequate for research in product development, the sample size of each sample - especially for sports optics and power tools - were limited. However, even this small sample size revealed high consistency regarding specific product development phenomena e.g. the fuzzy front-end or the high interactivity. The reason for the restricted evidence for the reliability of the constructs lies in the fact that the main purpose of the questionnaire was to compare different product developments. Adapting the questionnaire to address the reliability issues would have manipulated the comparability. Therefore, the improvement of the reliability is to be part of future research. All in all, the case study presented a solid contribution on aligning design phenomena quantitatively in industrial product development environments.

\section{References}

Andreasen, M.M., Hansen, C.T. and Cash, P. (2015), Conceptual Design, Springer International Publishing AG, Cham. https://doi.org/10.1007/978-3-319-19839-2

Baccarini, D. (1996), “The concept of project complexity”, International Journal of Project Management, Vol. 14 No. 4, pp. 201-204. https://doi.org/10.1016/0263-7863(95)00093-3

Clarkson, P.J. and Eckert, C. (2005), Design Process Improvement, Springer-Verlag, London. https://doi.org/10.1007/978-1-84628-061-0

Eder, W. and Hosnedl, S. (2010), Introduction to Design Engineering - Systematic Creativity and Management, CRC Press, Boca Raton, https://doi.org/10.1201/b10536

Ehrlenspiel, K. and Meerkamm, H. (2013), Integrierte Produktentwicklung, Carl Hanser Verlag, München. https://doi.org/10.3139/9783446436275 
Elgh, F. and Poorkiany, M. (2012), "Supporting Traceability of Design Rationale in an Automated Engineer-toOrder Business Model”, Proceedings of the DESIGN 2012 / 12th International Design Conference, Dubrovnik, Croatia, The Design Society, Glasgow, pp. 1425-1434.

Gopsill, J.A., McMahon, C. and Hicks, B. (2013), "The Communication Patterns of Engineers within SME in 2012", Proceedings of the 19th International Conference on Engineering Design (ICED13), Design for Harmonies, Vol.7: Human Behaviour in Design, Seoul, Korea, August 19-22, 2013, The Design Society, Glasgow, pp. 159-168.

Haberfellner, R., de Weck, O., Fricke, E. and Vössner, S. (2012), Systems engineering, Orell Füssli Verlag, Zurich. Hall, A.D. (1962), A Methodology for Systems Engineering, Princeton University Press,

Hashemi Farzaneh, H., Kaiser, M.K., Schröer, B., Srinivasan, V. and Lindemann, U. (2012), "Evaluation of Creativity - Structuring Solution Ideas Communicated in Groups Performing Solution Search", Proceedings of the DESIGN 2012 / 12th International Design Conference, Dubrovnik, Croatia, The Design Society, Glasgow, pp. 1871-1880.

Kallenborn, O. (2013), New Product Development as Complex Adaptive Socio-Technical Systems, PhD thesis, EBS Busines School.

Koehler, N., Naumann, T. and Vajna, S. (2014), "Supporting the Modeling of Traceability Information", Proceedings of the DESIGN 2014/ 13th International Design Conference, Dubrovnik, Croatia, The Design Society, Glasgow, pp. 1811-1820.

Koehler, N., Naumann, T. and Vajna, S. (2015), "Evaluating the Need for Traceability in Product Development: A Preliminary Study", Proceedings of the 20th International Conference on Engineering Design (ICED 15) Vol 5: Design Methods and Tools - Part 1, Milan, Italy, July 27-30, 2015, The Design Society, pp. 187-196.

Königs, S.F., Beier, G., Figge, A. and Stark, R. (2012), “Traceability in Systems Engineering”, Advanced Engineering Informatics, Vol. 26 No. 4, pp. 924 -940. https://doi.org/10.1016/j.aei.2012.08.002

Lindemann, U., Mauerer, M. and Braun, T. (2009), Structural complexity management, Springer-Verlag, Berlin. https://doi.org/10.1007/978-3-540-87889-6_1

Maier, A.M. and Störrle, H. (2011), "What are the Characteristics of Engineering Design Processes?", Proceedings of the 18th International Conference on Engineering Design (ICED 11), Impacting Society through Engineering Design, Vol. 1: Design Processes, Lyngby/Copenhagen, Denmark, August 15-19, 2011, The Design Society, Glasgow, pp. 188-198.

Maier, A.M., Kreimeyer, M., Hepperle, C., Eckert, C.M., Lindemann, U. and Clarkson, P.J. (2011), "Exploration in Correlations between Factors Influenceing Communication in Complex Product Development", Concurrent Engineering, Vol. 16 No. 1, pp. 27-59. https://doi.org/10.1177/1063293x07084638

Maurer, M.S. (2007), Structural Awareness in Complex Product Design, PhD thesis, Technische Universität München.

Naumann, T. (2016), “Theory of Social Systems Engineering”, Proceedings of the DESIGN 2016 14th International Design Conference, Dubrovnik, Croatia, The Design Society, Glasgow, pp. 45-56.

Naumann, T. and Koehler, N. (2014), "Meta-Model of Sociotechnical Systems: Derivation, Structure and Content, Tools and Methods of Competitive Engineering", International Symposium on Tools and Methods of Competitive Engineering (TMCE 2014), Budapest, Hungary.

Naumann, T., Königs, S., Kallenborn, O. and Tuttass, I. (2011), "Social Systems Engineering - An Approach for Efficient Systems Development", Proceedings of the 18th International Conference on Engineering Design (ICED 11), Impacting Society through Engineering Design, Vol. 4: Product and Systems Design, Lyngby/Copenhagen, Denmark, August 15-19, 2011, The Design Society, Glasgow, pp. 357-368.

Ouertani, M.Z., Baina, S., Gzara, L. and Morel, G. (2011), “Traceability and management of dispersed product knowledge during design and manufacturing", Computer-Aided Design, Vol. 43 No. 5, pp. 546-562. https://doi.org/10.1016/j.cad.2010.03.006

Patzak, G. (1982), Systemtechnik - Planung komplexer innovativer Systeme, Springer-Verlag, Berlin. https://doi.org/10.1007/978-3-642-81893-6

Ramesh, B. and Jarke, M. (2001), “Toward reference models for requirements traceability”, IEEE Transactions on Software Engineering, Vol. 27 No. 1, pp. 58-93. https://doi.org/10.1109/32.895989

Rieckher, J. (1871), Die Metaphysik des Aristoteles, Heimann, Stuttgart.

Storga, M. (2004), "Traceability in product development", Proceedings of DESIGN 2004, the 8th International Design Conference, Dubrovnik, Croatia, The Design Society, pp. 911-918.

Vajna, S. (2014), Integrated Design Engineering, Springer-Verlag, Berlin. https://doi.org/10.1007/978-3-64241104-5

Weber, C. (2005), "What is complexity?", Proceedings ICED 05, the 15th International Conference on Engineering Design, Melbourne, Australia, August 15-18, 2005, pp. 292-293. 
Winkler, S. and von Pilgrim, J. (2010), “A survey of traceability in requirements engineering and model-driven development", Software and Systems Modelling, Vol. 9 No. 4, pp. 529-565. https://doi.org/10.1007/s10270009-0145-0

Nico Koehler, Wirt.-Ing.

Otto von Guericke University Magdeburg, Information Technology and Mechanical Engineering Inselstr. 3, 35576 Wetzlar, Germany

Email: koehler.nico@me.com 